\title{
Exclusión del requisito de representación gráfica en el régimen marcario de la Comunidad Andina de Naciones
}

\section{Exclusion of the graphic representation requirement in the Andean Community of Nations' trademark regime}

María Emilia Torres Montenegro *

Recibido / Received: 24/05/2021

Aceptado / Accepted: 19/10/2021

DOI: $10.18272 /$ ulr.v8i2.2316

Citación:

Torres Montenegro, M.E. «Exclusión del requisito de representación gráfica en el régimen marcario de la Comunidad Andina de Naciones». USFQ Law Review, Vol 8, no 2, octubre de 2021, pp. 131 155, doi: $10.18272 /$ ulr.v8i2.2316.

Investigador independiente, casilla postal 17-1200-841, Quito 170901, Pichincha, Ecuador. Correo electrónico: emiliatorresmontenegro@gmail.com ORCID iD: https:/orcid.org/0000-0003-0046-609X 


\title{
RESUMEN
}

En el ámbito de la propiedad industrial, existen interpretaciones ambiguas respecto a la definición jurídica de una marca, las cuales al reconocer a la representación gráfica como un componente conceptual de la misma, le han dotado de un protagonismo innecesario a este elemento. Sin perjuicio de ello, es claro que el régimen marcario de la Comunidad Andina de Naciones define a las marcas únicamente a partir de su aptitud distintiva y exige a la representación gráfica únicamente como un requisito procedimental de su registro. Este requisito se fundamenta en el sistema atributivo, y su exigencia tiene sentido mientras permita determinar de una manera precisa el objeto de protección de las marcas. Ahora bien, la representación gráfica obstaculiza el registro de marcas no visuales que, a pesar de encajar en la definición de marca, no pueden ser representadas gráficamente, mas podrían ser representadas según las herramientas tecnológicas disponibles en la actualidad. Por ello, la representación gráfica se ha convertido en un requisito obsoleto, pues es incapaz de cumplir con su principal y esencial objetivo de precisión. Consecuentemente, el régimen marcario andino debe permitir la representación de cualquier forma, mediante la cual se permita al público en general determinar el objeto claro y preciso de la protección otorgada a su titular.

\section{Palabras Clave}

Comunidad Andina de Naciones; régimen marcario; registro de marcas; marcas no visuales; representación gráfica

\begin{abstract}
In the area of industrial property, there are ambiguous interpretations regarding the trademark's legal definition, in which, by recognizing the graphic representation as a conceptual component of it, this element has been given an unnecessary role. Notwithstanding this, the trademark regime of the Andean Community of Nations' clearly defines trademarks solely based on their distinctive aptitude and requires graphic representation only as a registration's procedural requirement. This requirement is based on the attributive system, and its requirement makes sense if it allows a precise determination of the trademark's object of protection. However, graphic representation hinders the registration of non-visual trademarks that, despite fitting the trademark's legal definition, cannot be graphically represented, but could be represented, according to the current available technology. Thus, graphical representation has become an obsolete requirement since it is incapable of fulfilling its main and essential objective of precision. Consequently, the Andean trademark regime must allow representation in any way that allows the public to precisely determine the trademark's object of protection granted to its owner.
\end{abstract}




\section{KEYWORDS}

Andean Community of Nations; trademark regime; trademark registration; non-visual trademarks; graphic representation

\section{INTRODUCCIÓN}

El marketing sensorial o sensory branding es una estrategia cada vez más utilizada por los operadores económicos a nivel mundial. ${ }^{1}$ Con el objetivo de atraer al público consumidor mediante todos los sentidos, los bienes y servicios que se ofertan en el mercado están siendo identificados no sólo por marcas visuales, sino también por marcas olfativas, sonoras, gustativas y táctiles. Un clásico ejemplo es el rugido del león antes de ver una película, el cual identifica a las producciones de la compañía estadounidense Metro-Goldwyn-Mayer Studios, Inc. Sin embargo, mientras el mercado se moderniza cada día gracias a los avances tecnológicos, la protección jurídica de este tipo de marcas no es una cuestión absolutamente pacífica.

Actualmente, en el régimen marcario nacional y en el de la Comunidad Andina (en adelante CAN), es discutible si las marcas no visuales reciben protección jurídica. Esto se desprende de la discordancia entre la amplitud del concepto legal de la marca y la exigencia de la representación gráfica como requisito procedimental para el registro, lo cual ha dado lugar a que doctrinarios y juristas realicen interpretaciones ambiguas al concepto legal de la marca. Como se detallará en los siguientes epígrafes, a pesar de que la definición legal de marca incluye a las marcas no visuales, ${ }^{2}$ el requisito para el registro de representación gráfica imposibilita la protección de este tipo de marcas.

La solución del problema es compleja en tanto algunos criterios jurisprudenciales han otorgado protagonismo a la representación gráfica al considerarla como un elemento idóneo y necesario dentro del derecho de marcas. Incluso, de manera equívoca, se la ha elevado como elemento conceptual de la marca. ${ }^{3}$ Sin embargo, a lo largo de este estudio se demostrará que, con base en los artículos 134 de la Decisión 486 sobre el Régimen Común sobre Propiedad Industrial de la CAN (en adelante Decisión 486) y 359 del Código Orgánico de la Economía Social de los Conocimientos (en adelante COESCCI); la representación gráfica es un mero requisito procedimental para el registro de

1 Blanca Torrubia Chalmeta, "El requisito de la representación gráfica: un límite de acceso al registro para las marcas no visuales", Actas de derecho industrial y derecho de autor, no. 32 (Enero 2012): 392.

2 Artículo 134, Decisión 486 sobre el Régimen Común sobre Propiedad Industrial de la Comunidad Andina de Naciones, [Decisión 486], RO 258 de 2 de febrero de 2001; Artículo 359, Código Orgánico de la Economía Social de los Conocimientos, [COESCCI], RO 899 de 9 de diciembre de 2016.

3 Interpretación Prejudicial No. 12-IP-2014, Tribunal de Justicia de la Comunidad Andina de Naciones, 14 de marzo de 2014, pág. 4; Interpretación Prejudicial No. 70-IP-2013, Tribunal de Justicia de la Comunidad Andina de Naciones, 8 de mayo de 2013, pág. 6; Interpretación Prejudicial No. 38-IP-2008, Tribunal de Justicia de la Comunidad Andina de Naciones, 10 de abril de 2008, pág. 5 . 
una marca. Por consiguiente, está bajo tela de duda si al ser un elemento puramente formal es indispensable dentro del régimen marcario, o si mas bien conviene analizar la idoneidad de mantener, eliminar o reformar este requisito del régimen marcario andino y nacional a fin de permitir el registro de marcas no visuales.

Así, el presente escrito analiza si el régimen marcario al que está sujeto el Ecuador puede acoplar lo dispuesto en la Unión Europea (en adelante UE), pues hasta el año 2015 este último régimen era muy similar al régimen andino y nacional con lo que respecta a la representación gráfica. Sin embargo, mediante el Reglamento No. 2015/2424 del Parlamento Europeo y del Consejo sobre la marca de la UE de 16 de diciembre de 2015 (en adelante Reglamento 2015/2424) se eliminó el requisito de representación gráfica de este régimen por considerar que un signo debe ser representado "[...] de cualquier forma que se considere adecuada usando la tecnología generalmente disponible [...]". ${ }^{4}$

De esta forma, en las líneas que siguen se argumentará por qué el régimen marcario andino y nacional debe acoplar su legislación a la realidad social y tecnológica del mercado y abandonar el requisito de representación gráfica. Esto, con el objetivo de que finalmente, las marcas no visuales puedan ser registradas sin obstáculos en el derecho marcario andino y nacional.

\section{LA REPRESENTACIÓN GRÁFICA ES UNA FIGURA FUNCIONAL}

A lo largo de esta sección se demostrará que contrario a lo señalado por ciertos sectores de la doctrina y la jurisprudencia, la definición de la marca contenida en la legislación andina y nacional excluye a la representación gráfica como elemento conceptual. En ese sentido, la representación gráfica se reduce a ser únicamente un requisito procedimental del registro que sirve para garantizar el sistema atributivo.

\subsection{INEXISTENCIA DE UN CRITERIO UNIFORME SOBRE LA NATURALEZA Y EFICACIA DE LA REPRESENTACIÓN GRÁFICA}

La representación de la marca es un elemento comunmente presente en las normativas de propiedad industrial, más aún en aquellos regímenes sujetos al sistema atributivo de registro. Sin embargo, existen varias posturas respecto de este elemento, sobre todo en el ámbito de su naturaleza y la eficacia de su exigencia, lo cual explica la importancia de este estudio.

4 Exposición de Motivos (9), Reglamento (UE) 2015/2424 del Parlamento Europeo y del Consejo de 16 de diciembre de 2015, Parlamento y Consejo de la Unión Europea, Diario Oficial de la Unión Europea No. L 341 de 24 de diciembre de 2015. 
En lo que se refiere a su naturaleza, además de ser un requisito procedimental del registro, la representación gráfica ha sido entendida mayoritariamente como un elemento conceptual de la marca. Así, Dámaso Ruiz-Jarabo Colomer $^{5}$, en el ámbito de la UE, y Manuel Guerrero Gaitán, ${ }^{6}$ en el ámbito de la CAN, coinciden en que para que cualquier signo constituya una marca, debe tener aptitud distintiva y ser susceptible de representación gráfica. Al contrario, estudiosos de la materia tales como el autor Sergio Balaña, excluyen acertadamente a la representación gráfica como elemento conceptual de la marca al señalar que este concepto debe estar ligado únicamente a la aptitud distintiva en tanto la representación gráfica es un mero requisito formal. ${ }^{7}$

$\mathrm{Al}$ respecto, en algunas interpretaciones prejudiciales, el Tribunal de Justicia de la CAN (en adelante TJCA) ha reconocido a la representación gráfica como elemento conceptual de la marca, ${ }^{8}$ mientras que en otros pronunciamientos se ha limitado a mencionar a la aptitud distintiva como único componente conceptual. ${ }^{9}$ Esto refleja la ambigüedad en torno al concepto legal de la mar$\mathrm{ca}$, pues incluso la autoridad judicial del régimen marcario al que pertenece Ecuador, no ha determinado de una manera clara y explícita la naturaleza de la representación gráfica, lo cual explica por qué la discusión alrededor de la exclusión de la representación gráfica del régimen marcario continúa siendo un tema actual y relevante.

No obstante lo anterior, queda claro que de cualquier manera la representación gráfica es un requisito procedimental para el registro, pues lo que se discute es si además de ser una exigencia meramente procedimental, dicho elemento también es un componente conceptual de la marca. En ese sentido, y respecto a la eficacia actual de la exigencia de la representación gráfica como requisito procedimental, la mayoría de autores coinciden en la dificultad de que las marcas no visuales cumplan con el mismo. Por un lado, y desde hace ya varios ańos, doctrinarios como Carlos Fernández Novoa anticipaban y proponían el uso alternativo de medios indirectos — como la fórmula químicapara que marcas olfativas, puedan cumplir con la literalidad de la representación gráfica. ${ }^{10}$

5 Dámaso Ruiz-Jarabo Colomer, "Conclusiones del Abogado General Sr. Dámaso Ruiz-Jarabo Colomer presentadas el 3 de abril de 2003”, en Caso-283/01 Tribunal de Justicia de la Unión Europea (Unión Europea: 2003), párr. 25.

6 Manuel Guerrero Gaitán, Derecho de Marcas. Teoria y Práctica Internacional. (Bogotá: Universidad Externado de Colombia, 2019), 206.

7 Sergio Balañá Vicente, "El entorno digital ¿̨Segunda oportunidad para la marca olfativa? Estudio acerca de la capacidad del signo olfativo para funcionar como marca en el mercado", Actas de derecho industrial y derecho de autor, no. 26 (2005-2006): 26.

8 Interpretación Prejudicial No. 12-IP-2014, Tribunal de Justicia de la Comunidad Andina de Naciones, 14 de marzo de 2014, pág. 4; Interpretación Prejudicial No. 70-IP-2013, Tribunal de Justicia de la Comunidad Andina de Naciones, 8 de mayo de 2013, pág. 6; Interpretación Prejudicial No. 38-IP-2008, Tribunal de Justicia de la Comunidad Andina de Naciones, 10 de abril de 2008, pág. 5 .

9 Interpretación Prejudicial No. 421-IP-2019, Tribunal de Justicia de la Comunidad Andina de Naciones, 18 de junio de 2020, párr. 1.3 .

10 Carlos Fernández-Nóvoa, Tratado Sobre Derecho De Marcas, (Madrid, Marcial Pons, 2004). p. 44 
Por otro lado, doctrinarios como José Manuel Otero Lastres, sostienen que en lugar de forzar la representación gráfica de las marcas no visuales, la ley debe permitir que el signo distintivo sea representado únicamente mediante reproducciones que sean perceptibles por los sentidos de la vista y del oído, hasta que no existan los avances tecnológicos que permitan realizar representaciones perceptibles por medio de los sentidos del olfato y del gusto. ${ }^{11}$

Con una visión un poco más evolutiva, autores como Blanca Torrubia Chalmeta, manifiestan que se debería permitir una mayor flexibilidad en los medios de representación. Así, acorde al régimen marcario europeo actual, propone que el solicitante pueda exponer una representación perceptible por cualquiera de los sentidos, siempre y cuando la reproducción sea clara, precisa, completa en sí misma, fácilmente accesible, inteligible, duradera y objetiva. ${ }^{12}$ En el mismo sentido, Manuel Guerrero Gaitán señala dentro del ámbito andino, que "[...] llegó la hora de abandonar, al igual que lo hizo la UE, ese apellido, el gráfico, en el requisito de representación [...]”. ${ }^{13}$

De lo expuesto se evidencia que no existe un criterio uniforme respecto de la naturaleza y eficacia de la representación gráfica, pues como se ha señalado se discute el rol de este elemento en el régimen marcario. Así también, y a partir de lo anterior, es polémico si su exclusión garantizaría o perjudicaría los derechos del titular y los del público en general. Sin embargo, como se detallará en los siguientes epígrafes, la solución a este problema inicia con una interpreatación literal de la legislación nacional y regional.

\subsection{LA APTITUD DISTINTIVA ES PER SE SUFICIENTE PARA QUE UN SIGNO CONSTITUYA UNA MARCA}

A la luz del régimen marcario andino y nacional, la distintividad es el elemento esencial para que un signo constituya una marca. Esto se desprende de la literalidad de la redacción de los artículos 134 de la Decisión 486 y 359 del COESCCI. Además, se refleja en la lista numerus apertus incluida en el mismo articulado y en las funciones de la marca.

Como bien lo ha señalado la doctrina más autorizada, "[...] la Ley proporciona al intérprete un valioso instrumento que le permite determinar los signos que pueden constituir una marca”. ${ }^{14}$ Así, para efectos de esclarecer el concepto

11 José Manuel Otero Lastres, "La representación de la marca en las propuestas comunitarias", Actas De Derecho Industrial Y Derecho De Autor, no. 33 (2013): 428.

12 Torrubia, "El requisito de la representación gráfica: un límite de acceso al registro para las marcas no visuales", 415.

13 Manuel Guerrero Gaitán, "Marcas no tradicionales y el requisito de representación gráfica”, en Seminario Internacional Gratuito de Propiedad Intelectual organizado por Instituto Iberoamericano de Leyes y Economía y Facultad de Jurisprudencia de la Universidad de Cuenca (Cuenca: Facultad de Jurisprudencia de la Universidad de Cuenca, 26 al 30 de octubre de 2020).

14 Fernández Novoa, Tratado Sobre Derecho De Marcas, 36. 
comunitario andino, resulta procedente traer a colación el primer inciso de los artículos 134 de la Decisión 486 y 359 del COESCCI. Ambos establecen que la marca es "[...] cualquier signo que sea apto para distinguir productos o servicios en el mercado. Podrán registrarse como marcas los signos susceptibles de representación gráfica". ${ }^{15}$ En ese sentido, además de la referencia al signo, los artículos en mención señalan dos figuras importantes: la aptitud distintiva y la susceptibilidad de representación gráfica.

Por un lado, el TJCA ha definido a la primera como la capacidad del signo para distinguir productos o servicios en el mercado y diferenciarlos de sus competidores. ${ }^{16}$ En esa misma línea, José Carlos Tinoco ha manifestado que la aptitud distintiva se refiere a que la marca "[...] tenga cuño propio y preciso, tomando fácil su reconocimiento y revelándose inconfundible con otras, aplicadas a productos o mercancías de procedencia diversa" ${ }^{17}$ Por otro lado, la representación gráfica hace mención a la idoneidad del signo para "[...] ser descrito o reproducido en palabras, imágenes, fórmulas u otros soportes, es decir, en algo perceptible para ser captado por el público consumidor". ${ }^{18}$

Si bien los artículos objeto de análisis hacen referencia a la aptitud distintiva y a la susceptibilidad de representación gráfica, se debe considerar que como lo ha manifestado el TJCA, el legislador ha plasmado en dichos artículos tanto la noción conceptual como los requisitos de procedimiento para el registro de una marca. ${ }^{19}$

En ese marco, la literalidad del enunciado "[p]odrán registrarse como marcas los signos susceptibles de representación gráfica" (énfasis añadido) ${ }^{20}$ tiene un efecto trascendental, pues en tanto la aptitud distintiva es la esencia del concepto de marca, la representación gráfica es únicamente un requisito procedimental para el registro, ya que no está incluido en la primera sección del artículo, en donde se define a la marca. En consecuencia, no cabe atar a la representación gráfica al concepto de marca ya que como bien lo ha señalado Sergio Balaña, "[...] este [debe] pivotar únicamente en torno al requisito esencial de la aptitud para diferenciar". ${ }^{21}$

La interpretación sería diferente si la redacción de los artículos en cuestión fuera igual a la del derogado Reglamento del Consejo de la Unión Europea No. 207/2009 de 26 de febrero de 2009 (en adelante Reglamento 207/2009).

15 Artículo 134, Decisión 486; Artículo 359, COESCCI.

16 Interpretación Prejudicial No. 155-IP-2012, Tribunal de Justicia de la Comunidad Andina de Naciones, 29 de enero de 2013, 5 .

17 José Carlos Tinoco Soares, Tratado De Propiedad Industrial De Las Américas (Buenos Aires: Lexis Nexis, 2006), 70.

18 Interpretación Prejudicial No. 047-IP-2013, Tribunal de Justicia de la Comunidad Andina de Naciones, 3 de abril de 2013, 3 .

19 Interpretación Prejudicial No. 242-IP-2015, Tribunal de Justicia de la Comunidad Andina de Naciones, 24 de agosto de 2015, párr. 64

20 Artículo 134, Decisión 486; Artículo 359, COESCCI (énfasis añadido).

21 Balańa, "El entorno digital ¿̨Segunda oportunidad para la marca olfativa?" 25. 
El artículo 4 de dicho cuerpo sostenía que:

[p] odrán constituir marcas comunitarias todos los signos que puedan ser objeto de una representación gráfica [...] con la condición de que tales signos sean apropiados para distinguir los productos o los servicios de una empresa de los de otras empresas. ${ }^{22}$

De la literalidad del artículo se desprende que la UE exigía la aptitud distintiva y la representación gráfica como elementos necesarios para que un signo pueda ser conceptualizado como marca, pues su "[...] posición en la parte inicial de dichos artículos permite considerarla como un elemento esencial de la definición de la marca". ${ }^{23}$ Por el contrario, como se ha demostrado, en el régimen comunitario andino todo signo con aptitud distintiva es una marca, mas sólo aquella susceptible de representación gráfica podrá ser registrada.

A pesar de que esto se desprende de la literalidad de los artículos 134 de la Decisión 486 y 359 del COESCCI, y como se ha anticipado, en algunas ocasiones el TJCA ha incluido a la representación gráfica al momento de definir la marca. En ese sentido, el alto tribunal ha señalado que la marca es un

[...] bien inmaterial constituido por un signo [...] susceptible de representación gráfica que sirve para distinguir en el mercado productos o servicios, a fin de que el consumidor o usuario medio los identifique, valore, diferencie y seleccione sin riesgo de confusión o error acerca del origen o la calidad del producto o del servicio. ${ }^{24}$

$\mathrm{Al}$ vincular la representación gráfica al concepto de marca, esta definición concuerda con lo estipulado en el Reglamento 207/2009 de la UE, mas no con lo señalado en la legislación andina. Por ello, otras puntualizaciones del TJCA al definir una marca como "[...] un bien inmaterial que permite identificar o distinguir los diversos productos y servicios que se ofertan en el mercado" 25 o "[...] cualquier signo que sea apto para distinguir productos o servicios en el mercado", ${ }^{26}$ son precisas y afines a lo dispuesto en la legislación andina y nacional, pues acorde a la legislación, definen a la marca únicamente desde la aptitud distintiva del signo.

Como se mencionó, el hecho de que la aptitud distintiva es suficiente para que un signo constituya una marca, se refleja en dos aspectos: (i) en el enunciado

22 Artículo 4, Reglamento (CE) 207/2009 del Consejo de 26 de febrero de 2009, Consejo de la Unión Europea, Diario Oficial de la Unión Europea No. L 78/1 de 24 de marzo de 2009.

23 Torrubia, "El requisito de la representación gráfica," 414.

24 Interpretación Prejudicial No. 12-IP-2014, Tribunal de Justicia de la Comunidad Andina de Naciones, 14 de marzo de 2014, pág. 4; Interpretación Prejudicial No. 70-IP-2013, Tribunal de Justicia de la Comunidad Andina de Naciones, 8 de mayo de 2013, pág. 6; Interpretación Prejudicial No. 38-IP-2008, Tribunal de Justicia de la Comunidad Andina de Naciones, 10 de abril de 2008, pág. 5. (erronea citación- ver guía de autor)

25 Interpretación Prejudicial No. 23-IP-2014, Tribunal de Justicia de la Comunidad Andina de Naciones, 13 de mayo de $2014,5$.

26 Interpretación Prejudicial No. 421-IP-2019, Tribunal de Justicia de la Comunidad Andina de Naciones, 18 de junio de 2020, párr. 1.3 . 
no taxativo de signos que pueden constituir marcas y (ii) en las funciones de la marca. Primero, el enlistado numerus apertus al que se ha hecho referencia, incluye entre otros, a las palabras, imágenes, sonidos y los olores. ${ }^{27}$ Así, "[...] esta lista abierta cubre tantos signos visibles como no visibles [...]"28 en virtud de que "[e]l signo distintivo puede ser percibido por la vista, por el oído, por el tacto, por el olfato o, incluso, por el gusto". ${ }^{29}$ Seguidamente, se puede concluir que la capacidad distintiva de un signo se extralimita al sentido de la vista.

Lo anterior reafirma la necesidad de distinguir en los artículos 134 de la Decisión 486 y 359 del COESCCI el concepto de marca y los requisitos de procedimiento para el registro, pues ha quedado demostrado que los signos - visuales o no- con aptitud distintiva, encajan en el concepto de marca ya que la distintividad puede ser reflejada a través de cualquiera de los sentidos. Sin embargo, la representación gráfica se limita al sentido de la visión. ${ }^{30}$ Consecuentemente, la representación gráfica no debe ser considerada como un componente del concepto de marca, la cual al ser definida únicamente como signo con aptitud distintiva, va más allá del sentido de la vista.

Por otra parte, el que la aptitud distintiva sea el elemento conceptual esencial de la marca se refleja en las funciones de la marca, pues la capacidad del signo de cumplir con estas funciones viene dada únicamente por dicha aptitud. La doctrina ha manifestado que "[l]a verdadera y única función esencial de la marca es distinguir un producto o un servicio, de otros" ${ }^{31}$ Es decir, la función más importante es la distintiva en tanto, como la ha considerado el TJCA, "[1] as restantes funciones, [...] se encuentran subordinadas a la capacidad distintiva del signo, pues sin esta no existiría el signo marcario". ${ }^{32}$

Esto evidencia una vez más que la aptitud distintiva es el elemento esencial que constituye a la marca y que la representación gráfica no figura dentro de su concepto, ya que a falta de aquella, el signo sería incapaz de cumplir con la función indicadora de procedencia empresarial, indicadora de calidad, condensadora del goodwill y publicitaria.

\footnotetext{
Artículo 134, Decisión 486; Artículo 359, COESCCI.

28 Interpretación Prejudicial No. 242-IP-2015, párr. 66.

29 Dámaso Ruiz-Jarabo Colomer, "Conclusiones del Abogado General Sr. Dámaso Ruiz Jarabo Colomer, presentadas el 6 de noviembre de 2001”, en Asunto C-273/00, Tribunal de Justicia de la Unión Europea, (Unión Europea: 2001), párr. 22.

30 Juan José Arias, "La registrabilidad de las marcas no tradicionales", en Simposio sobre el régimen de propiedad industrial Comunidad Andina. Bogotá, Colombia: 2015. 10.

31 Jorge Otamendi, Derecho de marcas. (Buenos Aires: Abeledo Perrot, 2003), 9.

32 Interpretación Prejudicial No. 421-IP-2019, Tribunal de Justicia de la Comunidad Andina de Naciones, 18 de junio de 2020, párr. 1.6.
} 


\subsection{LA REPRESENTACIÓN GRÁFICA ES UN MERO REQUISITO PROCEDIMENTAL}

Como se ha dicho a lo largo de este trabajo, la representación gráfica es simplemente un "[...] requisito formal, objetivo y funcional por oposición a la aptitud diferenciadora o carácter distintivo que es un requisito de primer orden $[\ldots]^{\prime 33}$ al ser el elemento primordial que define la marca. Entonces, es tan solo un requisito procedimental del registro, que tiene su razón de ser en el sistema atributivo y que busca garantizar el funcionamiento de este último.

\subsubsection{LA REPRESENTACIÓN GRÁFICA SE FUNDAMENTA EN EL SISTEMA ATRIBUTIVO}

El sistema atributivo o registral está recogido en la legislación andina y nacional e implica que el derecho exclusivo sobre una marca se adquiere a partir de su registro ante la autoridad nacional competente. ${ }^{34}$ Por ende, sin registro el solicitante no podrá ejercer las facultades de explotar la marca, ejercer actos de disposición y de impedir que terceros, bien registren una marca idéntica o similar, o bien realicen determinados actos con su marca (ius prohibendi). ${ }^{35}$ Por este motivo, el registro tiene una importancia trascendental en el régimen marcario ecuatoriano, andino y continental, pues solamente con su concesión, los operadores económicos estarán legitimados para hacer oponible su signo respecto de los demás competidores en el mercado.

Si bien es cierto que con base en lo dispuesto en los Títulos X y XIII de la Decisión 486 y Capítulos X y XV del COESCCI, un nombre comercial o una marca notoriamente conocida podrían beneficiarse de una tutela por un procedimiento diferente al registro, ${ }^{36}$ es importante recalcar que se trata de excepciones. Luego, no hay duda sobre la magnitud del registro para los efectos jurídicos de la marca en general.

Ahora, la representación gráfica es únicamente una porción del registro. Por ello, se ha dicho que este requisito tiene su razón de ser en el sistema atributivo, pues su carácter funcional "[...] radica básicamente en la opción legislativa a favor del registro como vía fundamental para la adquisición de la marca $[\ldots] " .{ }^{37}$ En ese sentido, el requisito material en cuestión se exige en la primera

33 Balańa, "El entorno digital ¿̨Segunda oportunidad para la marca olfativa?" 25.

34 Artículo 154, Decisión 486; Artículo 364, COESCCI.

35 Interpretación Prejudicial No. 26-IP-2017, Tribunal de Justicia de la Comunidad Andina de Naciones, 1 de diciembre de 2017, párr. 1.2.

36 Artículo 191, Decisión 486; Artículo 229, Decisión 486; Artículo 416, COESCCI; Artículo 460, COESCCI.

37 Luis Alberto Marco Arcalá.. "Prohibiciones absolutas", en Comentarios a la Ley de Marcas, Alberto Bercovitz Rodríguez-Cano, (Navarra, Aranzadi, 2008). 139. 
etapa del proceso de registro, pues la solicitud de registro debe contener la representación gráfica de la marca. ${ }^{38}$

De lo expuesto se desprende que la representación gráfica tiene sentido en el régimen marcario porque es un requisito procedimental del registro. En consecuencia, "[...] debe quedar claro que, por importante que sea el requisito de la representación gráfica, únicamente puede tener relevancia en el marco del sistema registral" ${ }^{39}$ Por consiguiente, lo verdaderamente esencial no es el requisito de representación gráfica, sino el proceso de registro, pues según lo detallado, es el proceso sine qua non para adquirir los derechos marcarios.

\subsubsection{LA REPRESENTACIÓN GRÁFICA BUSCA GARANTIZAR EL FUNCIONAMIENTO DEL SISTEMA ATRIBUTIVO}

En virtud de que los derechos marcarios no tienen un ejercicio ilimitado, ${ }^{40}$ el registro debe establecer de manera precisa, el marco de protección de los derechos del titular. Esto debido a que como ha señalado el TJCA, la expresión exacta, completa y rigurosa de las marcas protege de mejor manera los derechos de los titulares de las marcas, permite a los operadores económicos determinar la competencia en el mercado así como evitar infracciones o conocer de las mismas y previene a los consumidores del riesgo de confusión o asociación..$^{41}$ Por ello, el sistema atributivo tiene como objetivo precisar la marca en el registro de tal manera que se garantice seguridad jurídica al titular, a los competidores en el mercado y a los consumidores.

En virtud de lo dicho, considerando que la representación gráfica tiene su razón de ser en el sistema atributivo, este requisito debe alcanzar el objetivo de precisión al que se ha hecho referencia en el párrafo anterior. En esa misma línea se ha pronunciado la doctrina al reconocer que el propósito de la representación gráfica es "[...] proteger y dar publicidad a su apropiación por un empresario, [...] con el objetivo de singularizar sus productos o los servicios que presta". ${ }^{42}$

Así, la exigencia de la representación gráfica es útil en el régimen marcario andino y nacional en la medida en que permita precisar la marca. Esto nuevamente evidencia que lo que el régimen marcario protege a cabalidad no es el requisito de representación gráfica en sí mismo, sino el sistema atributivo y por ende, el objetivo de precisar la marca.

38 Artículo 138, Decisión 486.

39 Torrubia, "El requisito de la representación gráfica," 414.

40 Interpretación Prejudicial No. 101-IP-2013, Tribunal de Justicia de la Comunidad Andina de Naciones, 22 de mayo de $2013,7$.

41 Interpretación Prejudicial No. 242-IP-2015, párr. 94.

42 Dámaso Ruiz-Jarabo Colomer, "Conclusiones del Abogado General Sr. Dámaso Ruiz Jarabo Colomer presentadas el 6 de noviembre de 2001,” párr. 38. 
Lo anterior también puede inferirse del criterio jurisprudencial del TJUE en el Caso Sieckmann. En este último, el alto tribunal señaló que la representación gráfica debe ser "[...] clara, precisa, completa en sí misma, fácilmente accesible, inteligible, duradera y objetiva" ${ }^{43}$

Fácilmente accesible e inteligible para que la generalidad de los interesados en consultar el registro, que son los otros productores y los consumidores, puedan aprehenderla. Clara, precisa y completa, para que se sepa, sin duda alguna, la indicación que se monopoliza. Duradera y objetiva, para que ni el paso del tiempo ni el cambio de destinatario afecten a la identificación o a la percepción del signo. ${ }^{44}$

De esa manera, el TJUE valida que la representación gráfica busca precisar la marca y garantizar el funcionamiento del sistema atributivo, pues las cualidades de claridad, integridad, accesibilidad, inteligibilidad, duración y objetividad califican a la precisión, mas no a la representación gráfica per se. De conformidad con esto, el TJCA manifestó acertadamente en el Caso Old Parr, que la representación gráfica es una expresión de la precisión en tanto las cualidades en mención son aspectos formales que la comportan. ${ }^{45}$

En definitiva y en relación con lo detallado en el anterior epígrafe, la representación gráfica no es un elemento conceptual de la marca. Al contrario, es un mero requisito procedimental dentro de la etapa del registro que encuentra su fundamento en el sistema atributivo, y por ende busca garantizar su funcionamiento. Por ello este requisito material tiene utilidad dentro del régimen marcario andino y nacional en tanto sea capaz de precisar el registro de la marca a fin de garantizar seguridad jurídica para el titular, los operadores económicos y los consumidores.

\section{ES IDÓNEO ELIMINAR EL LÍMITE GRAMATICAL GRÁFICO DE LA REPRESENTACIÓN GRÁFICA}

En los siguientes epígrafes se evidenciará que la representación gráfica es un requisito obsoleto, pues es incapaz de precisar el registro de marcas no visuales. En consecuencia, resulta idóneo eliminar el límite gramatical gráfico de dicha exigencia, ya que la mera representación de la marca permite mayor precisión, lo cual garantiza el funcionamiento del sistema atributivo. 


\subsection{El REQUISITO DE REPRESENTACIÓN GRÁFICA TRABA EL REGISTRO DE MARCAS NO VISUALES}

Según lo evidenciado en los anteriores epígrafes, la Decisión 486 reconoce que el único elemento esencial dentro del régimen marcario es la distintividad mientras que la representación gráfica es un mero requisito procedimental. Sin embargo, la realidad dista de esta postura, pues algunos criterios jurisprudenciales y las oficinas nacionales competentes han otorgado a la representación gráfica el carácter de indispensable.

De ahí que de forma similar a lo que sucedía con el régimen marcario de la $\mathrm{UE}^{46}$, en el régimen de la CAN existe actualmente una vulneración al derecho a la seguridad jurídica, pues si bien se reconoce que las marcas no visibles encajan en el concepto jurídico de marca, la exigencia del requisito de representación gráfica obstruye cualquier protección jurídica a través del registro. Esto debido a que, como se advirtió anteriormente, la representación gráfica está ligada a una percepción estrictamente visual. En cambio, las marcas no visibles son perceptibles por sentidos distintos a la vista.

Como se detallará a continuación, los signos no visuales difícilmente pueden ser precisados por medio de una representación gráfica que reproduzca de forma exacta el objeto de protección de este tipo de marcas. Es por ello que la representación gráfica es un requisito obsoleto e ineficaz, pues al no poder precisar el registro de las marcas no visuales, es incapaz de cumplir con el objetivo para el que fue creado: garantizar el funcionamiento del sistema atributivo.

\subsubsection{Problemática de la Representación gráfica en las MARCAS SONORAS}

En el año 2003, el TJUE dentro del Caso-283/01 (Caso Shield Mark) definió a las marcas sonoras como aquellos signos capaces de "[...] distinguir los productos o los servicios de una empresa de los de otras empresas y que puedan ser objeto de representación gráfica" ${ }^{47} \mathrm{Al}$ contrario, acorde a lo señalado en los artículos 134 de la Decisión 486 y 359 del COESCCI, cabe recordar que dentro del régimen marcario andino y nacional, una marca sonora es aquel signo o medio con aptitud distintiva que se percibe por el sentido del oído. Por ejemplo, marcas sonoras son aquellas "[...] melodías, jingles o simples sonidos que, inconscientemente, nuestras mentes asocian con algún producto o servicio en particular". ${ }^{48}$

46 Exposición de Motivos (9), Reglamento 2015/2424.

47 Caso C-283/01, Tribunal de Justicia de la Unión Europea, 27 de noviembre de 2003, párr. 41.

48 Baldo Kresalja Rosselló, "La registrabilidad de las marcas auditivas, olfativas y las constituidas por color único en la Decisión 486 de la Comunidad Andina de Naciones", en XIX Congreso Internacional de la Asociación lnteramericana de Propie- 
En lo que respecta a la registrabilidad de estas marcas, si bien el TJCA no ha emitido ningún pronunciamiento, el TJUE señaló en el caso ya mencionado la aptitud del pentagrama para ser una representación gráfica clara, precisa, completa en sí misma, fácilmente accesible, inteligible, duradera y objetiva. ${ }^{49}$ Así también, la doctrina ha considerado que otros medios para representar gráficamente las marcas sonoras son el oscilograma, espectro sonoro, espectrograma, sonograma y la descripción. ${ }^{50}$

Respecto a la última, la doctrina ha alegado que no es una representación precisa en tanto adolece de vaguedad y claridad. ${ }^{51} \mathrm{Al}$ igual que sucede en las marcas olfativas, la descripción puede resultar en una representación gráfica útil en tanto el consultante conozca el sonido al que se hace referencia. De ahí que a fin de cumplir con el objetivo de precisión del sistema atributivo, la descripción no resulta suficiente.

En relación al pentagrama, oscilograma, espectro sonoro, espectograma y sonograma; el uso de dichas herramientas para representar gráficamente las marcas sonoras presentan un gran obstáculo. Si bien no existe normativa o jurisprudencia que señale que el público debe percibir el signo de forma inmediata, ${ }^{52}$ no es menos cierto que el uso de estos medios implica una carga injustificada sobre el consultante para poder percibir el signo de una manera precisa. Esto, debido a que se necesita del conocimiento de un experto para poder interpretar los sonidos a través de estos medios y compararlos con otros existentes en el mercado. ${ }^{53}$

En relación con ello, si bien “(...) los esfuerzos se han concentrado en encontrar las formas más adecuadas de representación de estos signos, de manera que no resulten demasiado complejas o gravosas para los solicitantes o las oficinas (...)", ${ }^{44}$ el TJCA ha reconocido que "[...] la problemática de la representación gráfica del signo a los fines de su publicación (y posibles oposiciones) atañe a la Oficina de marcas [...]". ${ }^{55}$ De ahí que el uso de estos medios para representar gráficamente las marcas sonoras, impide el correcto funcionamiento del sistema atributivo, pues la precisión del objeto de protección de la marca sonora se sustenta en un esfuerzo excesivo del consultante que no está obligado a soportar.

50 Organización Mundial de la Propiedad Intelectual, "Marcas no tradicionales", Revista de la OMPI, no. 1. (Febrero 2009): 5.

51 Dámaso Ruiz-Jarabo Colomer, "Conclusiones del Abogado General Sr. Dámaso Ruiz Jarabo Colomer presentadas el 3 de abril de 2003”, párr. 43.

52 Caso C-283/01, párr. 44.

53 Arias, "La registrabilidad de las marcas no tradicionales," 13.

54 Gretell Leyva Salazar, "Un acercamiento a las marcas animadas”, Revista La Propiedad Inmaterial, no. 24. (julio -diciembre 2017): 11, DOI: https://doi-org.ezbiblio.usfq.edu.ec/10.18601/16571959.n24.01.

55 Interpretación Prejudicial No. 242-IP-2015, párr. 75. 


\subsubsection{Problemática de la Representación gráfica en las MARCAS OLFATIVAS}

El TJUE estableció en el Caso Sieckmann que un olor puede constituir una marca si además de tener aptitud distintiva, es susceptible de representación gráfica. ${ }^{56}$ En cambio, de conformidad con la Decisión 486 y el COESCCI, en el régimen andino un olor podrá considerarse como marca en tanto tenga aptitud distintiva, pues "[...] si la representación gráfica no figura en el concepto legal de marca, [...] el olor, aunque no fuera representable gráficamente, sería conceptualmente una marca $[\ldots] " .57$

Ahora, frente a los signos distintivos sonoros, las marcas olfativas encuentran un obstáculo más grande en la representación gráfica. A pesar de que el TJCA tampoco ha desarrollado esta cuestión, el TJUE estableció en el Caso Sieckmann que "[...] si bien no son perceptibles en cuanto tales por la vista, sí pueden resultar visibles mediante una representación gráfica” ${ }^{58} \mathrm{Al}$ respecto, cabe señalar que dicha afirmación atenta contra la precisión protegida por el sistema registral, pues la percepción visual de un signo no visual impide un entendimiento integral del mismo.

Por esa razón la misma autoridad judicial determinó que una fórmula química, la descripción por medio de palabras y el depósito de una muestra del olor, no son representaciones gráficas claras, precisas, completas en sí mismas, fácilmente accesibles, inteligibles, duraderas y objetivas. ${ }^{59}$ En virtud de ello, el Caso Sieckmann valió más por lo que negó que por lo que afirmó, pues si bien motivó por qué las representaciones del solicitante no eran acordes a la ley, no señaló de qué manera se puede representar gráficamente una marca olfativa ${ }^{60}$

En lo que se relaciona al depósito de la muestra de olor, el TJUE negó su aplicación prima facie debido a que no es un método gráfico, que además no es estable ni duradero. ${ }^{61}$ Con lo que respecta a la descripción, la autoridad judicial impuso que el uso de palabras para detallar un signo de este tipo no es lo suficientemente claro, objetivo y preciso. ${ }^{62}$

$\mathrm{Al}$ respecto, en algunos casos la descripción sí puede funcionar como representación gráfica. ${ }^{63}$ Empero, al igual que en las marcas sonoras, esto se subor-

\footnotetext{
56 Asunto C-273/00, Tribunal de Justicia de la Unión Europea, 12 de diciembre de 2002. párr. 45.

57 Otero, "La representación de la marca en las propuestas comunitarias", 422.

58 Asunto C-273/00, párr. 32.

59 Ibid., párr. 73.

60 Otero, "La representación de la marca en las propuestas comunitarias", 420.

61 Asunto C-273/00, párr. 71.

62 Ibid., párr 70.

63 Solicitud No. 1241420 de mayo de 2008 ante Oficina de Propiedad Intelectual de Australia. "OMPI Base Mundial de Datos Sobre Marcas.”. Organización Mundial de Propiedad Intelectual. (Octubre 2020). https://www3.wipo.int/branddb/ es/\#.
} 
dina a que el público en general tenga con anterioridad una referencia sobre el olor al que se hace alusión. ${ }^{64}$ De ahí que si se describe, por ejemplo, el olor a eucalipto, el público en general podrá percibir el aroma. En cambio, si se hace referencia a un aroma creado específicamente para identificar un producto o un servicio - tal y como acontece en el Caso Sieckmann- el público en general no podrá entenderlo y por tanto la descripción resultaría inútil.

En relación a la fórmula química, el TJUE señaló que, además de que no representa el olor si no la sustancia per se, esta no es suficientemente inteligible para el público en general, en tanto "[...] pocas personas serían capaces de reconocer, en una fórmula de ese tipo, el olor en cuestión". ${ }^{55}$ Este pronunciamiento genera varias dudas ya que en este caso, el TJUE coincide con el TJCA en que no debe existir una carga injustificada en el consultante para determinar la naturaleza exacta de la marca a partir del registro. Sin embargo, al mismo tiempo, genera inseguridad jurídica ya que como se señaló anteriormente, la misma autoridad judicial reconoció en el Caso Shield Mark, que el pentagrama — que podría considerarse como la fórmula química de la marca sonora- sí tiene aptitud para ser una representación gráfica.

Esta incertidumbre ha suscitado varias críticas, pues para algunos autores, admitir como representación gráfica al pentagrama pero negar la fórmula química, supone una discriminación injustificada ${ }^{66}$ De esta forma, se evidencia la falta de criterios uniformes en el régimen de protección jurídica de las marcas no visibles y su representación gráfica.

En ese sentido, a partir de las negaciones dispuestas en el Caso Sieckmann, se ha propuesto la posibilidad de representar gráficamente un signo distintivo olfativo a través del análisis cromatográfico. ${ }^{67}$ Lastimosamente, al igual que la fórmula química, el uso de esta herramienta impone un esfuerzo injustificado en terceros no familiarizados con su uso, lo cual desvirtuaría el sistema atributivo. Esto, a partir de que se pondría en riesgo la posibilidad de que los operadores económicos se opongan a las solicitudes de registro "[...] por no ser capaces de identificar si el signo solicitado infringe algún derecho individual o colectivo". ${ }^{68}$

En definitiva, a partir de lo señalado se desprende que no existe certeza sobre qué representación gráfica permitiría al público en general determinar el objeto exacto de protección de una marca olfativa de manera que se garantice el funcionamiento del sistema atributivo.

64 Arias, "La registrabilidad de las marcas no tradicionales," 12.

65 Asunto C-273/00, párr. 69.

66 Torrubia, "El requisito de la representación gráfica," 415.

67 Camilo Andrés Díaz Trillos, "La registrabilidad del olor en Colombia, una posibilidad marcaria para el Siglo XXI”, Jurídicas CUC 13, no. 1 (Enero - Diciembre 2017): 62, http://dx.doi.org/10.17981/juridcuc.13.1.2017.3.

68 Arias, "La registrabilidad de las marcas no tradicionales", 12. 


\subsubsection{Problemática de la Representación gráfica en las mar- CAS TÁCTILES}

Al igual que las marcas sonoras y olfativas, las táctiles deben ser definidas como los signos o medios perceptibles por medio del tacto, con aptitud para distinguir productos o servicios y diferenciarlos de sus competidores. Así también lo ha reconocido la International Trademark Association desde 2006. ${ }^{69}$

Respecto a la registrabilidad de este tipo de marcas, el TJCA desarrolló esta cuestión en el Caso Old Parr. En esta interpretación prejudicial, la autoridad judicial reconoció que la representación gráfica ejerce un rol importante en las marcas no tradicionales ${ }^{70}$ pues la descripción y la fotográfia de la marca táctil permiten reconocer distintas características de la marca. ${ }^{71}$

A pesar de ello, del ratio decidendi del caso, se desprende que si bien la representación gráfica de la marca táctil es útil, no es per se suficiente para, de conformidad con el sistema atributivo, dar a conocer de manera exacta el objeto de protección de una marca táctil. Por esto, el TJCA admitió que para una mayor precisión del objeto de la marca táctil,“[...] se debe contar con varias representaciones de la marca, tomadas en perspectivas diferentes y según distintos métodos y modalidades". ${ }^{72}$ De ahí que del análisis expuesto por el TJCA, se concluye que a fin de dar cumplimiento al sistema registral, las representaciones gráficas de una marca táctil no permiten determinar con exactitud el objeto de protección.

\subsubsection{Problemática de la Representación gráfica en las MARCAS GUSTATIVAS}

Las marcas gustativas son, entre las marcas no visibles, las menos comunes. Esto se debe a que generalmente el sabor es una característica de los productos, lo cual anula la aptitud distintiva de este tipo de signos. ${ }^{73}$ Pese a ello, mientras un signo que se perciba por medio del gusto pueda distinguir un producto o servicio y diferenciarlo de sus competidores, podrá constituir una marca en el régimen marcario andino y nacional.

$\mathrm{Al}$ igual que en las marcas olfativas y sonoras, no existe desarrollo jurisprudencial por parte del TJCA que aclare las cuestiones de registrabilidad de este tipo de marcas. No obstante, el TJUE en el Caso R 120/2001-2 (en adelante

69 Interpretación Prejudicial No. 242-IP-2015, párr. 56.

70 Ibid., párr. 96.

71 Juan Manuel Indacochea, "La interpretación prejudicial 242-IP-2015 del Tribunal Andino", Revistas IUS ET VERITAS, no. 56. (Julio 2018): 121, http://doi.org/10.18800/iusetveritas.201801.007

72 Interpretación Prejudicial No. 242-IP-2015, párr. 98.

73 Torrubia, "El requisito de la representación gráfica," 407. 
Caso Eli Lilly), determinó que si la descripción de una marca olfativa no es una representación gráfica clara, precisa, completa en sí misma, fácilmente accesible, inteligible, duradera y objetiva; entonces la descripción de una marca gustativa tampoco cumple con estos requisitos. ${ }^{74}$

De la misma forma que en el Caso Sieckmann, el TJUE no especificó en el Caso Eli Lilly de qué manera una marca gustativa podría cumplir con la literalidad de la representación gráfica, por lo que no existe certidumbre ante la manera de representar gráficamente este tipo de marcas de conformidad con el sistema atributivo.

\subsection{LA REPRESENTACIÓN PER SE GARANTIZA PRECISIÓN EN EL SISTEMA ATRIBUTIVO}

Como ha quedado plasmado, la representación gráfica no permite precisar las marcas no visibles, lo cual obstaculiza el registro de estas marcas. Sin embargo, al mismo tiempo es menester que la marca se reproduzca de alguna forma a fin de cumplir con el sistema registral. Por ello, en aras de garantizar el funcionamiento de dicho sistema, y al igual que lo hizo la UE, el régimen marcario andino y nacional debe excluir el límite gramatical gráfico de las representaciones de las marcas. Esto, de manera que las marcas sonoras, olfativas, táctiles y gustativas se reproduzcan por medios — claros, precisos, completos en sí mismos, inteligibles y duraderos y objetivos- que sean perceptibles por cualquiera de los cinco sentidos del ser humano.

El TJCA coincide con esta posición, pues otorgando preferencia al desempeño del sistema registral por sobre el carácter funcional del requisito de representación gráfica, ha reconocido que se debe admitir las "[...] representaciones de un signo distintas a las gráficas cuando éstas sean más aptas para identificarlo" ${ }^{75}$

\subsubsection{EL USO DE REPRESENTACIONES GRÁFICAS, AUDITIVAS O MIXTAS PERMITE PRECISAR LAS MARCAS SONORAS}

Sin duda, a pesar de que las marcas sonoras tienen más facilidad para reproducirse de manera visual, ha quedado claro que la representación gráfica no es suficiente e impone una carga injustificada sobre el público en general, lo cual obstaculiza la precisión, y por ende el funcionamiento del sistema atributivo. Empero, la apertura al uso de representaciones no gráficas o la combinación de éstas con representaciones gráficas, permite precisar las marcas de manera que se garantice el sistema registral.

74 Caso R 120/2001-2, Tribunal de Justicia de la Unión Europea, 4 de agosto de 2003, párr. 12.

75 Interpretación Prejudicial No. 242-IP-2015, párr. 87. 
Esta última posición ha sido respaldada a nivel mundial. Por ejemplo, cabe hacer referencia al Tratado de Singapur sobre el Derecho de Marcas (en adelante Tratado de Singapur), el cual no ha sido ratificado por Ecuador, pero sí por 51 países alrededor del mundo, entre los que está Perú, país miembro de la CAN. Este Tratado "[...] constituye en la actualidad la referencia fundamental en el ámbito de las marcas atípicas, no convencionales, no-tradicionales o nuevos tipos de marcas [...]" ${ }^{76}$ Su Reglamento señala que la reproducción de una marca sonora podrá consistir en una "[...] notación en un pentagrama, o en una descripción del sonido que constituye la marca, o en una grabación analógica o digital de ese sonido, o toda combinación de lo anterior" ${ }^{77}$

Asimismo, la Organización Mundial de Propiedad Intelectual recomienda que, además de una representación gráfica, las marcas sonoras puedan cumplir con la exigencia de reproducción mediante "[...] una descripción textual de ese sonido, una grabación de ese sonido o cualquier combinación de esos elementos" ${ }^{78}$ Del mismo modo, en el ámbito de la UE, el Reglamento de Ejecución 2018/626 de la Comisión de la UE de 5 de marzo de 2018 (en adelante Reglamento 2018/626), acepta que se pueden utilizar archivos de audio como representación de la marca sonora. ${ }^{79}$

De hecho, la Superintendencia de Industria y Comercio de Colombia — país miembro de la CAN - sugiere que además de representarse gráficamente por medio de pentagramas, fonogramas, onomatopeyas, las marcas sonoras deben acompañarse de un archivo con la grabación digital del sonido. ${ }^{80}$ Es decir, la oficina nacional competente de Colombia reconoce que, contrario a lo estipulado en la ley, y con miras a precisar de mejor manera la marca sonora, es necesario que la representación gráfica de aquella se complemente con reproducciones auditivas. Incluso, reproducción auditiva del signo no representa un esfuerzo excesivo ni para el solicitante ni para la oficina de marcas, toda vez que actualmente las solicitudes de registro se presentan de manera electrónica, lo cual facilita el "[...] suministrar la reproducción del sonido en un fichero electrónico, por ejemplo, MP3 o.WAV, que puede ponerse a disposición de las partes interesadas o del público en la propia Oficina o su sitio Web". ${ }^{81}$

76 Juan Manuel Indacochea Jáuregui, "La marca táctil o de textura en el ámbito de la Comunidad Andina: A propósito de la interpretación prejudicial 242-IP-2015 del Tribunal Andino, Inconveniencias jurídicas y políticas de la actuación colombiana”, EAFIT: Journal of International Law 7, no. 1. (Enero - Junio 2016): 120.

77 Regla 3, Reglamento del Tratado de Singapur sobre el Derecho de Marcas, Singapur, 27 de marzo de 2006.

78 Recomendaciones para la gestión electrónica de las marcas sonoras, Norma ST. 68, Comité de Normas de la Organización Mundial de la Propiedad Intelectual, 24 de marzo de 2016, párr 5.

79 Artículo 3, Reglamento de Ejecución (UE) 2018/626 de la Comisión de 5 de marzo de 2018, Comisión de la Unión Europea, Diario Oficial de la Unión Europea No. L 104/37 de 24 de abril de 2018.

80 Superintendencia de Industria y Comercio, Manual de Marcas (Colombia: Superintendencia de Industria y Comercio, 2016), 31.

81 Comité Permanente sobre el Derecho de Marcas, Diseños Industriales e Indicaciones Geográficas de la Organización Mundial de la Propiedad Intelectual (OMPI), "Marcas no tradicionales: enseñanzas destacadas", en Decimoctava sesión, ed. Secretaría de la OMPI (Ginebra: OMPI, 2007), 5. 
El pronunciamiento de las oficinas de marcas refleja la inminente necesidad de un cambio legislativo, no sólo en el ámbito de las marcas sonoras sino para las marcas no visuales en general. De ahí que resulta importante recordar que el derecho pretende llegar a un consenso que se adapta a las necesidades de una realidad " [...] que se encuentra permanentemente in fieri y demanda por ello un esfuerzo continuo de renovación por parte de todos los operadores jurídicos, que es tanto como decir todos los individuos que componen una sociedad dada". 82

De lo dicho se desprende que el uso de representaciones no gráficas o la combinación de éstas con medios gráficos "[...] puede ser preferible a la simple representación gráfica si ello permite una identificación más precisa e [...] incrementa la seguridad juridica" ${ }^{83}$ De esta manera, queda a discreción del solicitante utilizar los medios gráficos o auditivos que crea convenientes a fin de reproducir la marca sonora de la manera más precisa posible.

Igualmente, cada oficina nacional competente debe estudiar si la representación gráfica, auditiva, o mixta, es lo suficientemente clara, precisa, completa en sí misma, fácilmente accesible, inteligible, duradera y objetiva para permitir a los operadores económicos y consumidores determinar el marco de protección de la marca sonora bajo análisis. En ese sentido, el Grupo Volkswagen ha registrado marcas sonoras utilizando únicamente pentagramas ${ }^{84} \mathrm{o}$ grabaciones de sonido. ${ }^{85}$ Asimismo, Land Business S.A. registró en Costa Rica una marca sonora utilizando exclusivamente la descripción “¡URRIA, URRIA!” ${ }^{86}$ Esto demuestra que no todos los registros son precisados de igual forma, pues el tipo de reproducción será lo suficientemente preciso o impreciso dependiendo de las circunstancias de cada caso.

En definitiva, es claro que la posibilidad de representar una marca sonora a través de medios auditivos permite ganar en precisión en el registro de este tipo de marcas. Esto trae como consecuencia el funcionamiento integral del sistema atributivo, pues finalmente los consultantes del registro podrán precisar los signos auditivos de manera inmediata y sin una carga injustificada.

82 Alejandro Nieto y Tomás-Ramón Fernández, El Derecho y El Revés (Barcelona: Editorial Ariel, S.A., 2006), 27.

83 Torrubia, "El requisito de la representación gráfica," 414.

84 Registro No. DE302020012407 de 15 de julio de 2020 ante la Oficina Alemana de Marcas y Patentes. "OMPI Base Mundial de Datos Sobre Marcas”. Organización Mundial de Propiedad Intelectual. (Octubre 2020). https://www3.wipo. int/branddb/es/\#.

85 Registro No. DE302019020963 de 10 de diciembre de 2019 ante la Oficina Alemana de Marcas y Patentes. "OMPI Base Mundial de Datos Sobre Marcas”. Organización Mundial de Propiedad Intelectual. (Octubre 2020). https://www3.wipo. int/branddb/es/\#.

86 Registro No. 171608 de 26 de noviembre de 2007 ante el Registro Nacional de Costa Rica. "OMPI Base Mundial de Datos Sobre Marcas”. Organización Mundial de Propiedad Intelectual. (Octubre 2020). https://www3.wipo.int/branddb/es/\#. 


\subsubsection{El USO DE REPRESENTACIONES PALPABLES PERMITE PRECISAR LAS MARCAS TÁCTILES}

Cómo se señaló anteriormente, el TJCA desarrolló la registrabilidad de la marca táctil en el Caso Old Parr. De ahí que el ente judicial determinó en esa interpretación prejudicial la manera en que la marca táctil debe cumplir con una representación clara, precisa, completa en sí misma, fácilmente accesible, inteligible, duradera y objetiva. Ahora bien, de manera descuidada el TJCA denotó que se debe hacer una interpretación amplia del requisito de representación gráfica, incluyendo como elemento de aquella a la muestra física de la marca táctil..$^{87}$

Esto significó un uso desacertado de palabras, pues la muestra física no es una representación gráfica. Al contrario, si bien el público en general puede ver la muestra física, la forma de percibir el signo - para propósitos de determinar el marco de protección del signo distintivo- será estrictamente táctil. En todo caso, no cabe duda de que el TJCA reconoció que la marca táctil debe ser representada también con una muestra física.

$\mathrm{Al}$ respecto, Manuel Guerrero Gaitán sostiene que la muestra física o depósito presenta varios problemas. Ello a partir de que las oficinas nacionales competentes en materia de propiedad intelectual han hecho grandes esfuerzos por digitalizar los procesos de registro y el hecho de que exista una obligación de revisar este depósito anula dicho esfuerzo. ${ }^{88}$ En consecuencia, el autor considera que se crea un problema logístico-administrativo, y una carga injustificada en el consultante. ${ }^{89}$

Sin embargo, la muestra física es una representación precisa que a diferencia de lo que sucede con, por ejemplo, el pentagrama para la marca sonora o la fórmula química para la marca olfativa, permite al consultante percibir el signo de manera directa e inmediata. Así, resulta ser una representación que cumple con el objetivo de precisión interpuesto por el sistema atributivo.

Por consiguiente, si bien hubiese sido más preciso que el TJCA determine que la representación gráfica de este tipo de signos puede ser complementada por una representación táctil como la muestra física, el criterio del tribunal es extremadamente claro. Así, es imperioso que la representación gráfica de la marca táctil se complemente con una reproducción palpable a fin de precisar la marca de manera que el consultante perciba el signo de manera integral.

87 Interpretación Prejudicial No. 242-IP-2015, párr. 101.

88 Guerrero, "Marcas no tradicionales y el requisito de representación gráfica”.

89 Ibid. 


\subsubsection{LA REPRESENTACIÓN PER SE PERMITE EL REGISTRO DE MARCAS OLFATIVAS Y GUSTATIVAS}

Como se demostró, la dificultad de representar gráficamente de manera precisa las marcas olfativas y gustativas es la más evidente. Ahora, cabe reconocer que con dicha dificultad no se restringe a las representaciones gráficas. Esto debido a que si bien actualmente se desarrollan tecnologías que, por ejemplo, permiten percibir olores de forma digital $^{90}$, en los regímenes marcarios no existe certeza sobre qué medios no gráficos podrían utilizarse para la representación precisa de marcas olfativas o gustativas. ${ }^{91}$

En ese sentido, existe una posición restrictiva y otra flexible respecto a la representación de las marcas olfativas y gustativas. En relación a la primera, doctrinarios como Otero Lastres defienden la postura restrictiva al manifestar que mientras no se concreten los avances tecnológicos, las reproducciones de las marcas deben limitarse a representaciones visuales y auditivas con el objetivo de garantizar seguridad jurídica. ${ }^{92}$

Respecto a esta postura es importante denotar que el problema no quedaría solventado. Esto debido a que como se ha reiterado en este análisis, las marcas olfativas y gustativas forman parte del concepto marcario - incluso se incorporan en la lista numerus apertus ${ }^{93}$ - pero la protección jurídica se desvanecería al momento de exigir únicamente representaciones gráficas o visuales. En tanto estos últimos medios no permitirían percibir el signo olfativo o gustativo de manera integral, el problema no quedaría zanjado.

En lo concerniente a ello, Manuel Guerrero Gaitán señala que si bien es válido sostener que las interpretaciones de la norma no deben ser abiertas a fin de garantizar seguridad jurídica, la postura detallada en el párrafo anterior presenta otro problema adicional. La contrariedad se relaciona con el hecho de que tanto en la UE como en la CAN, no es sólo un país el que decide, por lo que reformar las normas es un proceso que implicaría no sólo tiempo y recursos adicionales, sino el consenso de todos los países miembros. ${ }^{94}$ Así, Guerrero Gaitán defiende que, si se presenta la oportunidad de reformar la Decisión 486 , se debe sacrificar un poco de seguridad jurídica para darle un espacio de evolución al derecho. ${ }^{95}$

90 Cristina Fernández Cámara, "Marcas olfativas, 'sensory branding' y Reglamento UE 2015/2424”, Cuadernos de Derecho y Comercio, no. 67 (2017): 152.

91 José Barreda, "Algunas anotaciones respecto de los requisitos de registrabilidad de signos según la Decisión 486 de la Comunidad Andina", en Estudio de Derecho y Propiedad Intelectual. Homenaje a Arturo Alessandri Besa ed. Rodrigo Velasco, Marcos Morales (Santiago: Editorial Jurídica de Chile, 2011), 238.

92 Otero, "La representación de la marca en las propuestas comunitarias", 427.

93 Artículo 134, Decisión 486; Artículo 359, COESCCI.

94 Guerrero, "Marcas no tradicionales y el requisito de representación gráfica".

95 Ibid. 
Lo anterior es precisamente el fundamento de la postura flexible. El mejor ejemplo de esta segunda postura es la UE, pues como se ha reiterado, desde la vigencia del Reglamento 2015/2424 el régimen comunitario europeo sostiene que las marcas pueden ser representadas "[...] de manera que permita a las autoridades competentes y al público en general determinar el objeto claro y preciso de la protección otorgada a su titular" ${ }^{96}$ Así también, esta postura ha sido respaldada por el Tratado de Singapur, el cual estipula que ante la solicitud de registro de las marcas no visibles y no sonoras, se "[...] podrá exigir una o más representaciones de la marca, una indicación del tipo de marca y detalles relativos a la marca, según lo disponga la legislación de esa Parte Contratante". ${ }^{77}$

A diferencia de la postura restrictiva, esta última garantiza seguridad jurídica por dos razones. Primero, porque además de considerarlas conceptualmente como marca, la legislación permite la representación de las marcas olfativas y gustativas. Así, se accede a que el solicitante represente la marca de cualquier forma que se considere adecuada usando la tecnología generalmente disponible. ${ }^{98}$ Esto implica que el registro de marcas olfativas y gustativas depende solamente de que las oficinas nacionales competentes se doten de la tecnología necesaria para tal efecto. ${ }^{99}$

Segundo, esta postura sí garantiza seguridad jurídica debido a que "[...] el hecho de permitir una mayor flexibilidad en los medios de representación no debe pasar por alto la idea básica que subyace en la lista de criterios enunciados en Sieckmann [...]". ${ }^{100}$ En consecuencia, y tal como lo señala el Reglamento 2018/626, independientemente de la representación que se use para representar las marcas olfativas o gustativas, esta debe necesariamente ser lo suficientemente clara, precisa, completa en sí misma, fácilmente accesible, inteligible, duradera y objetiva. ${ }^{101}$ Esto, con el objetivo de que, como bien lo estipula el Reglamento No. 2017/1001 del Parlamento Europeo y del Consejo de 14 de junio de 2017 sobre la marca de la UE, las autoridades competentes y el público en general puedan determinar con claridad y exactitud el objeto preciso de la protección otorgada al titular, ${ }^{102}$ impulsando así, el funcionamiento del sistema atributivo.

Además, la adopción de la postura flexible incentiva el desarrollo tecnológico de los operadores y apertura la posibilidad de que exista un análisis jurídico en las oficinas de marcas así como un desarrollo jurisprudencial por parte del

96 Artículo 4, Reglamento 2015/2424.

97 Regla 3, Tratado de Singapur sobre el Derecho de Marcas, Singapur, 27 de marzo de 2006.

98 Exposición de motivos (9), Reglamento 2015/2424.

99 Balańa, "El entorno digital ¿̨Segunda oportunidad para la marca olfativa?” 27.

100 Torrubia, "El requisito de la representación gráfica," 415.

101 Artículo 3, Reglamento 2018/626.

102 Artículo 4, Reglamento (UE) 2017/1001 del Parlamento Europea y del Consejo de 14 de junio de 2017, Parlamento y Consejo de la Unión Europea, Diairo Oficial de la Unión Europea No. L 154/2 de 16 de junio de 2017. 
TJCA de las representaciones de las marcas olfativas y gustativas. De lo contrario, independientemente de que existan avances tecnológicos que permitan representar este tipo de marcas, al no ser representaciones estrictamente visuales o sonoras, estas no podrían ser conocidas por las autoridades competentes.

\section{Conclusiones}

En medio de la era digital es esencial otorgar protección jurídica a las marcas no visuales, las cuales son uno de los resultados de los grandes avances tecnológicos. Sin embargo, como ha quedado verificado, actualmente la CAN y por consiguiente Ecuador, no permiten tutelar de una manera integral este tipo de signos distintivos. Peor aún, la falta de protección se debe simplemente a la exigencia de la representación gráfica. Así, a lo largo de este estudio, se ha demostrado que actualmente, el requisito en cuestión no encuentra razón alguna para permanecer vigente en un régimen marcario con sistema atributivo, como lo es la CAN.

En primer lugar, la exclusión de la representación gráfica no altera en lo más mínimo la esencia del régimen marcario al que está sujeto Ecuador. Esto a partir de que, como quedó detallado en los primeros epígrafes, la representación gráfica es un mero requisito procedimental. En ese sentido, la esencia de la marca no depende en lo absoluto de esta exigencia, pues para que un signo constituya una marca, basta que tenga aptitud distintiva. Por esto último, es irrefutable que los signos sonoros, olfativos, táctiles y gustativos pueden constituir marcas y que el problema para la protección de estos signos distintivos recae únicamente en la exigencia del requisito de representación gráfica.

En segundo lugar, es idóneo eliminar el requisito de representación gráfica de la legislación andina y nacional en virtud de que ha perdido su capacidad para cumplir su principal objetivo. Tal y como se constató, la exigencia de la representación gráfica tiene sentido mientras sea capaz de precisar los registros marcarios a fin de garantizar el funcionamiento del sistema atributivo. Sin embargo, la representación gráfica es una figura incapaz de delimitar de una manera precisa el objeto de protección de signos no visuales. En definitiva, lo único que hoy en día logra la representación gráfica es imponer cargas injustificadas en los terceros consultantes y brindar incertidumbre respecto de la imposibilidad de traducir algo no visual a un campo visual.

Además la razón por la que el régimen marcario andino y nacional no ha adoptado la postura flexible respecto a la representación de la marca — como si lo hizo la UE - está fuera de lógica en medio de la era digital, donde la tecnología brinda soluciones. Así, la idoneidad de la exclusión de la represen- 
tación gráfica es indiscutible en tanto la tecnología generalmente disponible permitirá al régimen marcario gozar de precisión.

Por todo lo anterior, en concordancia con el ánalisis expuesto, es conducente proceder con una reforma al régimen marcario de la CAN, por medio de la cual se excluya a la exigencia gráfica, del requisito de representación. Así se permitirá que las marcas — con independencia de que sean visuales o nosean precisadas mediante reproducciones claras, precisas, completas en sí mismas, fácilmente accesibles, inteligibles, duraderas y objetivas. Así, finalmente se garantizará el funcionamiento del sistema atributivo, pues operadores económicos, operadores jurídicos y los titulares podrán conocer el objeto de protección de los signos distintivos de una forma precisa.

En definitiva, se sugiere que los artículos 134 de la Decisión 486 y 359 del COESCCI sean reformados. Así, se recomienda que el primer inciso de los artículos en cuestión señale la siguiente fórmula: "A efectos de este régimen, constituirá marca cualquier signo que sea apto para distinguir productos o servicios en el mercado y diferenciarlos de sus competidores. Para ser registrados, los signos distintivos deberán ser objeto de una representación que permita a las autoridades competentes y al público en general, determinar el objeto claro y preciso de la protección otorgada a su titular”. 
